\title{
Primary immune thrombocytopenia in children
}

\author{
Agnieszka Książek, Tomasz Szczepański
}

Department od Pediatric Hematology and Oncology, Zabrze, Medical University of Silesia, Katowice, Poland

\section{ABSTRACT}

Primary immune thrombocytopenia (ITP) is usually a benign and self-limiting disease in children. Due to the complex pathogenesis involving different immunological processes, its course may be unpredictable. The goal of therapy is to prevent severe, dangerous bleeding, especially intracranial bleeding. In addition to purpura, i.e. ecchymoses and bruises on the skin, mucosal bleeding may occur, e.g. from nose, gums, excessive menstrual bleeding, as well as severe bleeding from the gastrointestinal or urinary tract. First-line therapy relies on glucocorticoids (GKS) or intravenous immunoglobulins (IVIgG). The next stage of treatment is the inclusion of other immunosuppressive drugs or cytotoxic drugs. The introduction of thrombopoietin receptor agonists (TPO-RAs) for the treatment of this disease has been the biggest progress over the past 20 years. TPO-RAs are treatment of choice for chronic ITP. Splenectomy is now reserved only for children with chronic ITP resistant to thrombopoietin receptor agonists..

KEY WORDS:

primary immune thrombocytopenia, children, treatment.

Primary immune thrombocytopenia (ITP) is an acquired autoimmune disease characterized by isolated thrombocytopenia - a reduction of blood platelets below $100 \times 10^{9} / 1$, after excluding other disorders or conditions leading to thrombocytopenia. Until recently, the disease was referred to as idiopathic thrombocytopenic purpura, but the name was changed due to the lack or slight symptoms of haemorrhagic diathesis in most patients and the proven immune background of the disease [1]. Primary immune thrombocytopenia is one of the most common haematological diseases in children, occurring at a frequency of 1.9-6.4 cases per 100000 children per year, most often in children aged 1-7 years $[1,2]$. Chronic ITP occurs at a frequency of 0.5 out of 100000 children per year [2]. The annual incidence of ITP in patients in Poland, assessed on the basis of the PLATE study conducted in the period from October 2007 to September 2008 in 42 centres, in which 1331 patients were involved, is 3.5/100 000 [3, 4]. The disease has a seasonal character - the most cases are found in autumn and winter, the least in summer [5].
Primary immune thrombocytopenia in children, in contrast to adults (in whom the disease usually has an insidious onset, without a history of haemorrhagic diathesis or a history of infection, often goes into chronic form, and its course is variable and unpredictable), usually has a sudden onset, preceded by most often benign viral infection or infectious disease (varicella, measles, rubella, mumps, infectious mononucleosis) or vaccination, especially with attenuated vaccines [6-9]. Some chronic infections, e.g. cytomegalovirus or parvovirus infection, may also trigger thrombocytopenia [10]. As a rule, ITP is a mild disease, with good prognosis, and with a short course, spontaneously disappearing in most children within a few days to several months. In $10-20 \%$ of cases it turns into a chronic form [6-9]. Helicobacter pylori infection is well documented as a causative agent of persistent or chronic thrombocytopenia. However, based on strong evidence from observational studies, the 2019 ASH guidelines for ITP in children recommend against routine testing for $H$. pylori in children with chronic

\section{ADDRESS FOR CORRESPONDENCE:}

Agnieszka Książek, Department od Pediatric Hematology and Oncology, Zabrze, Medical University of Silesia,

3 Maja 13-15, 41-800 Zabrze, Poland, e-mail: aksiazek@sum.edu.pl 
ITP [11-15]. The only vaccine that has been proven to be associated with ITP is the measles, mumps, and rubella vaccine (MMR); the pathophysiology is unknown, but anti-GPIIb/IIIa antibodies have been detected in some patients. It has been found that the probability of disease occurring after MMR vaccination is 2.6/100 000 (0.087$4 / 100000)$ vaccine doses, and therefore less than after natural rubella infection (it was estimated that the incidence of ITP after rubella is less than that seen after measles and averages 1/3000 people). The disease appears within 6 weeks of vaccination, it is usually mild, resolving within a few days or weeks (in $90 \%$ the disease goes away completely within 6 months; in less than $10 \%$ of cases it goes into a chronic form) $[7,8,16]$.

Depending on the duration of thrombocytopenia, ITP is classified as newly diagnosed (up to 3 months from diagnosis), persistent (3-12 months from the time of diagnosis), or chronic ( $\geq 12$ months from the time of diagnosis) $[1,17]$.

Depending on the risk of bleeding, a mild, moderate, and severe form of the disease is distinguished [1].

Diagnosis of ITP is made after excluding other causes of thrombocytopenia, based on interview, physical examination, and morphology with peripheral blood smear. In justified cases, diagnostic tests are performed to rule out the causes of the secondary ITP (e.g. hepatitis C, systemic lupus erythematosus, anti-phospholipid syndrome, human immunodeficiency virus). It is advisable to measure immunoglobulin levels to exclude common variable immunodeficiency or isolated IgA deficiency. In a patient's medical history, it is usually found that symptoms of haemorrhagic diathesis and infection appear within 24-48 hours [4].

In a child with typical clinical and laboratory symptoms (isolated thrombocytopenia, increased platelet volume, giant platelets) for whom no treatment is required, bone marrow aspiration biopsy is not necessary. The examination is indicated if the patient has additional clinical symptoms (e.g. bone pain, hepatomegaly, splenomegaly, lymphadenopathy not explained by other causes) and abnormal blood counts in other cell lines. In addition, it is recommended that the examination be performed in the case of poor response or lack of response to firstline treatment and before starting steroid therapy. If the patient does not increase their platelet count after 3-6 months, it is recommended that a bone marrow examination, and antinuclear and anti-phospholipid antibody tests are performed $[10,18]$. On the other hand, ASH guidelines from 2019 recommend that bone marrow examination is unnecessary in children and adolescents with typical features of ITP and in children who fail IVIG therapy. They suggest that bone marrow examination is also not necessary in similar patients prior to initiation of treatment with corticosteroids or before splenectomy. Also testing for antinuclear antibodies is not necessary in the evaluation of children and adolescents with suspected
ITP [15]. Anti-platelet antibodies to specific platelet glycoprotein (GP) antibodies are not recommended for routine diagnostics because they are not specific (they are also detected in non-immune thrombocytopenia) and are not detected in half of the patients $[4,10,19,20]$. There are also no indications for routine testing for Helicobacter pylori infection in children [4].

Patients with ITP may have the following clinical symptoms: ecchymosis, spontaneous bruising, prolonged bleeding after injury, and mucosal bleeding (e.g. epistaxis, serious bleeding from the gums, gastrointestinal tract, urinary tract, menorrhagia) [10]. Symptoms of haemorrhagic diathesis usually appear when the platelet count is below $50 \times 10^{9} / 1$. Only $3 \%$ of patients have symptoms such as epistaxis or gastrointestinal bleeding. Severe complications are more likely at platelet counts below $10 \times 10^{9} / 1$, while trauma can cause bleeding at a higher platelet count. The probability of intracranial bleeding ranges from 0.1 to $0.5 \%$, and the potential risk factors are: a decrease in the number of platelets below $10-20 \times 10^{9} / 1$, head injury, use of drugs adversely affecting platelet function (e.g. non-steroidal anti-inflammatory drugs), and arteriovenous malformations of the brain [6, 21-23].

Most ITP patients have highly active platelets that are capable of forming compensating platelet aggregates that will reduce platelet counts. An inability to activate platelets in a selected group of ITP patients may be partly responsible for the development of severe haemorrhage. Also, platelet-released microparticles with procoagulant activity may play a role in maintaining haemostasis in ITP and explain why most ITP patients may have very low platelet counts without major bleeding, because it has been observed that patients with higher levels of microparticles have had fewer bleedings [24].

Since 2010, new guidelines for ITP diagnostics and treatment have been in force, developed by an international group of experts in haematology, which take into account the emergence of new therapeutic options and the current knowledge about the pathophysiology of the disease, enabling a better understanding of the specifics of the disease. Due to the small number of available high-quality randomized controlled trials using standard therapies, the recommendations for therapeutic management are mainly based on the opinions of experts based on clinical experience, not scientific evidence [10].

Initially, it was claimed that the underlying cause of the disease was only the destruction of platelets by IgG-class antiplatelet antibodies that bind to platelet cell membrane antigens and then are destroyed by tissue macrophages in the reticuloendothelial system, mainly in the spleen. Antibodies are most often directed against platelet glycoprotein GPIIb/IIIa or/and GPIb/IX. Many patients produce different antibodies, which is attributed to the phenomenon of epitope spreading. As a result of accelerated destruction, platelet survival is significantly reduced, which should result in increased bone marrow 
production. However, increasing evidence suggests that thrombopoiesis is also compromised. Antiplatelet antibodies can bind to megakaryocytes, and their growth is inhibited by accelerated apoptosis, leading to their immaturity and reduced productivity. In addition, megakaryocytes may be surrounded by neutrophils and macrophages, suggesting an inflammatory response against these cells $[25,26]$. In over $50 \%$ of patients, antiplatelet antibodies are not detected, which may be due to technical reasons, binding of autoantibodies to megakaryocytes, or alternative causes of thrombocytopenia [9, 27]. In the absence of antiplatelet antibodies, immune thrombocytopenia may be associated with complex cell-mediated mechanisms, including abnormally activated helper $\mathrm{T}$ cells, regulatory T-cell deficiency, or platelet destruction by CD8+ reactive cytotoxic T cells [28]. Increasing evidence indicates the significant position of impaired regulatory T-lymphocyte function, while their different role is still unclear in patients achieving spontaneous remission and in patients with chronic ITP. Platelet reactive T-cells are directed primarily against the GPIIb/ IIIa antigen. When exposed to GPIIb/IIIa fragments, they stimulate the production of antibodies. It has been found that hidden epitopes can be exposed and detected by the immune system in certain circumstances, e.g. due to infection. Antigen-presenting cells, by expressing new peptides, start activating $T$ cells that recognize these additional platelet antigens, which may play an important role in initiating and perpetuating ITP. T-cell clones reacting with enigmatic epitopes may be negatively selected in the thymus. In addition, in the case of post-infectious thrombocytopenia, the immune response to infection may generate the production of antibodies that crossreact with antigens or platelet immune complexes by binding to platelet Fc receptors [9]. Studies have shown that the presence of anti-GPIb/IX antibodies is associated with resistance to intravenous immunoglobulin treatment, suggesting the possibility of direct cytotoxicity or complement fixation as a mechanism of platelet destruction [29]. Chronic ITP patients often have increased Th1/Th2 lymphocyte ratio, expansion of oligoclonal $\mathrm{T}$ lymphocytes, and current cytotoxic T cells against autologous plaques. The emergence of anti-platelet antibodies and anti-platelet cytotoxic T cells is a consequence of the loss of immune tolerance for self-antigens. Other T-cell abnormalities have also been found in ITP patients, including reduced peripheral blood activity and impaired immunosuppressive function of regulatory $\mathrm{T}$ cells CD25+CD4+FoxP3+ [30]. CD3+ T cells of patients with active disease have altered expression of genes associated with apoptosis and are much more resistant to dexamethasone-induced immunosuppression compared to normal lymphocytes $[9,31]$. Primary immune thrombocytopenia has also been proven to be associated with the accumulation and activation of $\mathrm{T}$ cells in the bone marrow by increasing the expression of VLA- 4 and CX3CR1. Such activated $\mathrm{T}$ cells can mediate the destruction of platelets in the bone marrow [32]. These findings suggest that autoreactive $\mathrm{T}$ cells in patients with active disease are not removed by normal apoptosis mechanisms, allowing further destruction of platelets. Regulatory T cells, which should inhibit autoreactive T-cell proliferation, are also quantitatively reduced in ITP patients, which further contributes to limiting normal T-cell function [33].

Thrombopoietin (TPO) is the basic regulator of normal thrombopoiesis. Endogenous TPO is produced in the liver and binds to receptors on megakaryocytes in the bone marrow, increasing their number, accelerating growth and maturation, and inhibiting apoptosis, thereby stimulating them to produce platelets. The circulating level of TPO is inversely proportional to the speed of platelet production: when platelet production is low, TPO levels increase; when platelet production is increased, the TPO level decreases. ITP patients have functional TPO deficiency, contributing to the state of thrombocytopenia. The level of thrombopoietin is normal or only slightly increased, suggesting that the lack of compensatory stimulation of megakaryocytes may contribute to reduced platelet production [34]. The introduction of thrombopoietin receptor agonists for the treatment of immune thrombocytopenia has been a major breakthrough in the treatment of this disease. They are the only group of drugs for which efficacy has been proven in randomized trials. They are a new treatment option for $2^{\text {nd }}$-and $3^{\text {rd }}$-line patients who have failed standard immunosuppressive therapy. Their mechanism of action is based on stimulating the bone marrow to produce platelets. They bind to thrombopoietin (TPO-R) receptors present on platelets and megakaryocytes and activate JAK2 and STAT5 kinase phosphorylation, which leads to increased thrombopoiesis. Their efficacy finally confirmed the new hypothesis of ITP aetiology, according to which peripheral platelet destruction is accompanied by inhibition of platelet-forming megakaryocyte function. These drugs mimic the action of endogenous thrombopoietin in the body, but because they have no structural similarity, they are not immunogenic. In addition, unlike other available therapies, they do not have immunosuppressive effects [34-38].

The main goal of ITP treatment is to obtain a safe number of blood platelets that inhibits bleeding or prevents bleeding while maintaining an acceptable quality of life of the patient and the least toxicity of treatment [1]. According to Polish recommendations, treatment is usually required in patients with platelets below $20-30 \times 10^{9} / 1$ and/or symptoms of haemorrhagic diathesis; it is rarely necessary when the platelet count exceeds $50 \times 10^{9} / 1$ when there are no other factors that increase the risk of bleeding [4]. International recommendations advise that children who do not have haemorrhagic symptoms should not start drug treatment regardless of the number of platelets, but instead use an expectant strategy [10]. 
Most children with mild to moderate clinical symptoms only require observation or supportive care (e.g. anti-fibrinolytic drugs, hormonal therapy in girls with heavy and prolonged menstruation) at an outpatient clinic and with a slight reduction in activity, waiting for spontaneous remission of the disease. Children with platelets above $20-30 \times 10^{9} / 1$ are usually stable with no clinical symptoms, so they can be followed without treatment. Some children (especially adolescents who are aware of their appearance) with a platelet count of $10-30 \times 10^{9} / 1$, who have skin symptoms may benefit from treatment by positively affecting their well-being. Drug therapy should also be considered in patients with moderate bleeding and an increased risk of bleeding. In the event of an exacerbation of symptoms of a haemorrhagic diathesis, a bed regime should be introduced.

Depending on the severity of bleeding, appropriate patient management is indicated:

- $1^{\text {st }}$ degree - minor bleeding, few petechiae $(<100)$, $\leq 5$ bruises $\leq 3 \mathrm{~cm}$ in diameter, no cutaneous and $\mathrm{mu}-$ cosal bleeding $\rightarrow$ observation,

- $2^{\text {nd }}$ degree - mild bleeding, many petechiae ( $\left.>100\right)$, $>5$ large bruises ( $>3 \mathrm{~cm}$ in diameter), no mucosal bleeding $\rightarrow$ observation or treatment of selected children,

- grade 3 - moderate bleeding, overt mucous bleeding, troublesome in the course of life $\rightarrow$ treatment in selected children,

- grade 4 - mucosal bleeding or suspected internal bleeding $\rightarrow$ treatment $[10]$.

The main clinical problem of the disease is an increased risk of bleeding despite the absence or presence of minimal symptoms of a haemorrhagic diathesis. Bleeding tendency does not correlate with platelet count. Based on the severity of cutaneous and mucosal symptoms, the risk of intracranial bleeding cannot be predicted; therefore, when qualifying for treatment of children, platelet count, level of activity, and lifestyle of the child, as well as psychosocial factors, should be taken into account [2]. Therapeutic management should be individual to each patient after taking into account the clinical condition, accompanying bleeding-related diseases, tolerance of adverse effects, and the expectations of the patient and his/her legal guardians [10, 39, 40]. In clinical practice, treatment decisions are often made with very low platelet counts, despite no significant bleeding, for fear of severe ITP complications and in response to parental expectations, as well as no serious side effects with short-term therapy [41].

The treatment of ITP uses drugs with different mechanisms of action. The recommended treatment in the majority of patients is the waiting attitude (observation of the child), which is effective in $50-77 \%$ of cases. The time to remission is from a few days to about 6 months.

The first line of treatment includes the following:

1) Intravenous immunoglobulins (IVIgG) - their mechanism of action is blocking anti-platelet antibodies.
They cause an increase in the number of platelets in $80 \%$ of cases; they are used in two schedules: administration of the drug at a dose of $0.4 \mathrm{~g} / \mathrm{kg}$ b.w. for 2-5 days or a single dose of $0.8-1 \mathrm{~g} / \mathrm{kg}$ b.w. with the option of repeat therapy (currently recommended). The most common side effect is headache, and rarely serious side effects such as kidney failure and thrombosis.

2) Glucocorticoids (GKS) - prevent the destruction of platelets by macrophages in the liver and spleen, increasing their number in the blood. An optional first-line drug is oral prednisone at a dose of $1-2 \mathrm{mg} / \mathrm{kg}$ b.w. for a maximum of 14 days or at a dose of $4 \mathrm{mg} / \mathrm{kg}$ b.w. for 3-4 days with a maximum daily dose not exceeding $180 \mathrm{mg}$. The method is effective in $72-8 \%$ of cases depending on the dose of the drug. Due to numerous side effects (mood disorder, aggression, reduced activity, gastritis, cushingoid obesity), which increase in proportion to the duration of use, the drug should be administered for as short a period as possible to achieve a safe platelet count. No response after 3-4 weeks indicates steroid resistance, at which point treatment should be terminated. If there is a need to use corticosteroids for at least 2 months in order to achieve a platelet value of at least $30 \times 10^{9} / 1$ and/or to relieve the symptoms of a haemorrhagic diathesis, steroid dependence is assumed.

The ASH 2019 guideline panel suggests corticosteroids rather than IVIG in children with newly diagnosed ITP, who have non-life-threatening mucosal bleeding and/or diminished quality of live due to significant cost associated with IVIG, the need for intravenous access, possible admission, and donor exposure with IVIG and the potential serious side effects (thrombosis and renal failure) compared with corticosteroids.

In the treatment of sudden and life-threatening bleeding combination therapy is recommended - intravenous administration of high doses of GKS with IVIgG. In addition, transfusion of platelet concentrate is often necessary. In exceptional cases, urgent splenectomy may be considered. In patients with heavy menstrual bleeding, antifibrinolytic drugs (Exacyl $20 \mathrm{mg} / \mathrm{kg}$ every 6-8 hours) and hormonal supplementation are additionally included. Antifibrinolytic therapy is also used for mucosal bleeding (except for urinary tract). Cyclonamine is recommended for all patients.

Patients whose initial treatment has not led to a permanent increase in platelet count are eligible for second-line treatment. The goal of treatment for persistent or chronic disease is to maintain a safe platelet count (above $50 \times 10^{9} / 1$ ) for haemostasis, which minimizes the risk of bleeding for the patient. Drugs as in first-line therapy are used, with the use of oral GKS for as short a period as possible.

When IVIgG or GKS treatment is ineffective, the following treatment options are available:

1) Dexamethasone at an oral dose of $28-40 \mathrm{mg} / \mathrm{kg}$ b.w./day for 4 days (1-4 cycles, every cycle lasts 14 or 
28 days) - the effectiveness is $80 \%$, but there may be serious side effects such as insomnia, aggressive behaviour, lack of concentration.

2) High doses of methylprednisolone - intravenously $30 \mathrm{mg} / \mathrm{kg}$ b.w./day for 3 days, then $20 \mathrm{mg} / \mathrm{kg}$ b.w./day for 4 days $-60-100 \%$ of patients respond to treatment, while the side effects are more severe than with prednisone. According to international recommendations, the drug is administered orally to children with persistent or chronic ITP.

3) Rituximab - a chimeric monoclonal antibody that binds to CD20 surface antigen present on B lymphocytes and acts immunosuppressively. Although the drug is not registered for ITP treatment, it has been included in the therapy of $2^{\text {nd }}$ and $3^{\text {rd }}$ lines of this disease. In a 1 stline ITP study, rituximab was shown to increase its efficacy when co-administered with dexamethasone. It is administered in a dose of $100 \mathrm{mg}$ or $375 \mathrm{mg} / \mathrm{m}^{2} /$ week for 4 weeks. The therapy is well tolerated; in most cases only mild and transient side effects were observed, although some patients developed serum sickness. In addition, there have been reports of the possibility of long-term adverse events such as progressive multifocal leukoencephalopathy. Adequate response to treatment is obtained in $31-79 \%$ of cases $[4,9,10,15,17,39,42]$.

4) Thrombopoietin receptor agonists (TPO-RAs): eltrombopag, romiplostim - bind and activate thrombopoietin receptors $(\mathrm{Mpl})$, do not contain homologous sequences with thrombopoietin, and effectively stimulate platelet production. They have recently been approved (eltrombopag in 2015, romiplostim in 2018) by the US Food and Drug Administration for the treatment of children with persistent or chronic ITP when $1^{\text {st }}$-line therapy has proved ineffective. Avatrombopag's ITP approval is limited to adults. Thrombopoietin receptor agonists are the only FDA-approved second-line treatments in paediatric ITP and offer high response rates without immunosuppression and durable responses at the cost of prolonged or indefinite treatment. The new American Society of Haematology guidelines favour TPO-RA over rituximab and splenectomy in the treatment of children with ITP [15]. Data indicate that both agents are efficacious and safe in children, but no randomized trial has directly compared them. In Poland, romiplostim and eltrombopag are registered in adults and children $\geq 1$ year old diagnosed with chronic (longer than 12 months) ITP, who have had an inadequate response to previous standard pharmacological treatment with ITP. On 1 March 2018, eltrombopag was reimbursed under the drug program "Eltrombopag in the treatment of paediatric patients with cITP" (B98), and on 1 September 2020, also romiplostim was included in the reimbursement list. The recommended initial dose of romiplostim, administered subcutaneously once weekly, is $1 \mu \mathrm{g} / \mathrm{kg}$, followed by the platelet count. The average therapeutic dose is $3-4 \mu \mathrm{g} / \mathrm{kg}$, with a maximum dose of $10 \mu \mathrm{g} / \mathrm{kg}$. The recommended initial dose of eltrombopag in children 1 to 5 years old is $25 \mathrm{mg}$ orally, and in children 6-17 years old and in most adults it is $50 \mathrm{mg} /$ day, with subsequent dose adjustment based on platelet count (maximum $75 \mathrm{mg} /$ day). In both cases, continuing therapy is necessary to maintain an elevated platelet count. These drugs do not work in states of sudden bleeding because of their delayed action. Studies conducted to date in the paediatric population have shown high efficacy of romiplostim and eltrombopag treatment, and good tolerability and safety of the drug. TPO receptor agonists have been proven to effectively increase platelet counts in both post-splenectomy patients and those with preserved spleen. Both romiplostim and eltrombopag have been shown to reduce bleeding complications. Most thromboembolic events associated with their use have been observed in patients with at least one additional risk factor for thrombosis. The use of both romiplostim and eltrombopag may increase the risk of bone marrow fibrosis, but the importance of this has not yet been established. The increase in the amount of reticulin fibres in the bone marrow is thought to be the result of stimulation of the thrombopoietin receptor, leading to an increase in the number of megakaryocytes in the bone marrow, which can then secrete cytokines. Long-term side effects of TPO receptor agonists are not yet known [43-46]. Both agents are associated with significant costs. The decision about which agent to use may be dictated by patient preference. Eltrombopag is a once-daily oral medication, but with dietary restrictions related to interaction with divalent cations. It may also lead to iron deficiency. Additionally, eltrombopag is metabolized in the liver. Because of hepatotoxicity, hepatic function needs close monitoring. Romiplostim has none of these disadvantages, but because of weekly subcutaneous injections it typically requires clinic visits for administration [47].

Moreover, in patients with refractory, symptomatic thrombocytopenia, a more intense immunosuppressive therapy is sometimes necessary - treatment with cyclosporin A, azathioprine or vinca alkaloids (vincristine, vinblastine) alone or in IVIgG/GKS polytherapy with a good response to treatment in $70 \%$ of patients $[4,9,10,17]$. Combination chemotherapy is recommended in some patients, including cyclophosphamide, prednisolone, vincristine, and azathioprine or etoposide. Danazol might be also used in refractory ITP, but due to adverse effects in the form of hirsutism and acne, it is rarely included in paediatric patients [4].

When ITP is prolonged for over 12 months, splenectomy remains to be considered $[3,4,10-14,16]$. Splenectomy is effective in about $70-80 \%$ of patients with a chronic form of the disease. However, due to possible serious side effects (haemorrhage, peripancreatic haematoma, diaphragmatic abscess, wound infection, pneumococcal infection, fever, sepsis, thrombosis, death), the decision to carry it out is usually made as a last resort. It is recommended that the procedure be 
postponed for at least 12 months after diagnosis, also because of the possibility of late spontaneous remission of the disease. Due to the fact that the risk of sepsis is $3 \%$ and persists throughout the patient's life, the procedure should be preceded by a series of preventive vaccinations (against pneumococci, group $\mathrm{C}$ meningococci and Haemophilus influenzae) and performed under antibiotic protection. Patients and their parents must be informed about the increased susceptibility to infection, the need for preventive vaccinations, prophylactic antibiotics prior to surgical procedures, and aggressive treatment of all infections. Response to treatment is obtained within 1-56 days (maximum 2 months) after surgery. In patients with ITP undergoing splenectomy, who have an increase in platelet count immediately after surgery, followed by a recurrence of thrombocytopenia, an additional spleen should be sought $[6,10,48]$. Laparoscopic surgery to remove the extra spleen should then be the first-line treatment, enabling at least temporary remission of the disease to be achieved [49]. With the advent of thrombopoietin receptor agonists, it is advised to postpone splenectomy in chronic ITP in children as long as the treatment response is maintained.

In addition, several clinical trials evaluate new drugs for chronic ITP treatment, such as the non-peptide thrombopoietin receptor agonist (avatrombopag), the spleen tyrosine kinase inhibitor (fostamatinib), monoclonal antibodies: human anti-CD40 ligand antibody (CD154, IDEC-131), anti-IL-2 receptor (daclizumab), anti-CD52 (alemtuzumab, Campath-1H), and others. Preliminary results are promising; if new drugs are approved for use in ITP patients, an improvement in the quality of life of patients can be expected due to the high efficiency and low incidence of toxicity, and thus less frequent use of older immunosuppressive therapies causing numerous side effects [50].

Resistant thrombocytopenia occurs when the following criteria (all) are met:

1. No response or loss of response after splenectomy.

2. The need for treatment (containing low doses of corticosteroids) to minimize the risk of bleeding.

3. Primary ITP confirmed by exclusion of factors causing thrombocytopenia and/or disorders occurring with thrombocytopenia [1].

The problem of resistant ITP affects a small number of children, but the available therapeutic options may not have the desired effect. The disease significantly impairs daily functioning and physical activity, primarily due to dangerous bleeding and the side effects of the drugs used. Therefore, research into the efficacy of thrombopoietin receptor analogues in the treatment of refractory ITP is a very important issue.

\section{DISCLOSURE}

The authors declare no conflict of interest.

\section{REFERENCES}

1. Rodeghiero F, Stasi R, Gernsheimer T, et al. Standardization of terminology, definitions and outcome criteria in immune thrombocytopenic purpura of adults and children: report from an international working group. Blood 2009; 113: 2386-2393.

2. Terrell DR, Beebe LA, Vesely SK, et al. The incidence of immune thrombocytopenic purpura in children and adults: A critical review of published reports. Am J Hematol 2009; 85: 174-180.

3. Zawilska K. Samoistna plamica małopłytkowa - skala problemu. Acta Haematologica Polonica 2009; 40: 843-849.

4. Zawilska K, Podolak-Dawidziak M, Chojnowski K, et al. Polskie zalecenia postępowania w pierwotnej małopłytkowości immunologicznej, opracowane przez Grupę ds. Hemostazy Polskiego Towarzystwa Transfuzjologów i Hematologów. Pol Arch Med Wewn 2010; 120 (supl.): 10-16.

5. Nugent DJ. Immune Thrombocytopenic Purpura of Childhood. Hematology Am Soc Hematol Educ Program 2006: 97-103.

6. British Committee for Standard in Haematology General Haematology Task Force (2003) Guidelines for the investigation and management of idiopathic thrombocytopenic purpura (ITP) in adults, children and in pregnancy. Br J Haematol 2003; 120: 574-596.

7. Cecinati V, Principi N, Brescia L, et al. Vaccine administration and the development of immune thrombocytopenic purpura in children. Human Vaccines \& Immunotherapeutics 2013; 9: 1158-1162.

8. Bertuola F, Morando C, Menniti-Ippolito F, et al. Association between drug and vaccine use and acute immune thrombocytopenia in childhood: a case-control study in Italy. Drug Saf 2010; 33: 65-72.

9. Stasi R. Pathophysiology and therapeutic options in primary immune thrombocytopenia. Blood Transfus 2011; 9: 262-273.

10. Provan D, Stasi R, Newland AC, et al. International consensus report on the investigation and management of primary immune thrombocytopenia. Blood 2010; 115: 168-186.

11. Kuwana M. Helicobacter pylori-associated immune thrombocytopenia: Clinical features and pathogenic mechanisms. World J Gastroenterol 2014; 20: 714-723.

12. Neefjes VM, Heijboer H, Tamminga RY. H. Pylori Infection In Childhood Chronic Immune Thrombocytopenic Purpura. Haematologica 2007; 92: 576.

13. Arnold DM, Bernotas A, Nazi I, et al. Platelet count response to $H$. pylori treatment in patients with immune thrombocytopenic purpura with and without $\mathrm{H}$. pylori infection: a systematic review. Haematologica 2009; 94: 850-856.

14. Stasi R, Sarpatwari A, Segal JB, et al. Effects of eradication of Helicobacter pylori infection in patients with immune thrombocytopenic purpura: a systematic review. Blood 2009; 113: 1231-1240.

15. Neunert C, Terrell DR, Arnold DM, et al. American Society of Hematology 2019 guidelines for immune thrombocytopenia. Blood Adv 2019; 3: 3829-3866.

16. O’Leary ST, Glanz JM, McClure DL, et al. The Risk of Immune Thrombocytopenic Purpura After Vaccination in Children and Adolescents. Pediatrics 2012; 129: 248-255.

17. Provan D, Newland AC. Current Management of Primary Immune Thrombocytopenia. Adv Ther 2015; 32: 875-887.

18. Jubelirer SJ, Harpold R. The role of the bone marrow examination in the diagnosis of immune thrombocytopenic purpura: case series and literature review. Clin Appl Thromb Hemost 2002; 8: 73-76.

19. Brighton TA, Evans S, Castaldi PA, et al. Prospective evaluation of the clinical usefulness of an antigen-specific assay (MAIPA) in idiopathic thrombocytopenic purpura and other immune thrombocytopenias. Blood 1996; 88: 194-201.

20. Maślanka K, Sikorska A, Uhrynowska M, Konopka L. Autoprzeciwciała przeciwpłytkowe skierowane przeciw glikoproteinom 
płytek krwi GPIIb/IIIa i GPIb, a przebieg choroby i wyniki leczenia w małopłytkowościach samoistnych. Acta Haematol Pol 2006, 37: 241-248.

21. Neunert CE, Buchanan GR, Imbach P, et al. Severe hemorrhage in children with newly diagnosed immune thrombocytopenic purpura. Blood 2008; 112: 4003-4008.

22. Psaila B, Petrovic A, Page LK, et al. Intracranial hemorrhage (ICH) in children with immune thrombocytopenia (ITP): study of 40 cases. Blood 2009; 114: 4777-4783.

23. Neunert C, Noroozi N, Norman G, et al. Severe bleeding events in adults and children with primary immune thrombocytopenia: a systematic review. J Thromb Haemost 2015; 13: 457-464.

24. Neunert CE. Individualized treatment for immune thrombocytopenia: predicting bleeding risk. Semin Hematol 2013; 50: 55-57.

25. McMillan R, Wang L, Tomer A, et al. Suppression of in vitro megakaryocyte production by antiplatelet autoantibodies from adult patients with chronic ITP. Blood 2004; 103: 1364-1369.

26. McMillan R, The pathogenesis of chronic immune thrombocytopenic purpura. Semin Hematol 2007; 44 (4 Suppl 5): 3-11.

27. Cines DB, Bussel JB, Liebman HA, Luning Prak ET. The ITP syndrome: pathogenic and clinical diversity. Blood 2009; 113: 65116521.

28. Chow L, Aslam R, Speck ER, et al. A murine model of severe immune thrombocytopenia is induced by antibody- and CD8+ T cel mediated responses that are differentially sensitive to therapy. Blood 2010; 115: 1247-1253.

29. Go RS, Johnston KL, Bruden KC. The association between platelet autoantibody specificity and response to intravenous immunoglobulin $\mathrm{G}$ in the treatment of patients with immune thrombocytopenia. Haematologica 2007; 92: 283-284.

30. Nishimoto T, Kuwana M. CD4+CD25+Foxp3+ regulatory T cells in the pathophysiology of immune thrombocytopenia. Semin Hematol 2013; 50: 43-49.

31. Olsson B, Andersson PO, Jacobsson S, et al. Disturbed apoptosis of T-cells in patients with active idiopathic thrombocytopenic purpura. Thromb Haemost 2005; 93: 139-144.

32. Olsson B, Ridell B, Carlsson L, et al. Recruitment of T cells into bone marrow of ITP patients possibly due to elevated expression of VLA-4 and CX3CR1. Blood 2008; 112: 1078-1084.

33. Nugent D, McMillan R, Nichol JL, Slichter SJ. Pathogenesis of chronic immune thrombocytopenia: increased platelet destruction and/or decreased platelet production. Br J Haematol 2009; 146: 585-596.

34. Kuter DJ, Gernsheimer TB. Thrombopoietin and platelet production in chronic immune thrombocytopenia. Hematol Oncol Clin N Am 2009; 23: 1193-1211.

35. Bussel JB, Buchanan GR, Nugent DJ, et al. A randomized, double-blind study of romiplostim to determine its safety and efficacy in children with immune thrombocytopenia. Blood 2011; 118: 28-36.

36. Vishnu P, Aboulafia DM. Long-term safety and efficacy of romiplostim for treatment of immune thrombocytopenia. J Blood Med 2016; 7: 99-106.

37. Bussel JB, Hsieh L, Buchanan GR, et al. Long-term use of the thrombopoietin-mimetic romiplostim in children with severe chronic immune thrombocytopenia. Pediatr Blood Cancer 2015; 62: 208-213

38. Garzon AM, Mitchell WB. Use of thrombopoietin receptor agonists in childhood immune thrombocytopenia. Front Pediatr 2015; 3: 70.

39. Neunert C, Lim W, Crowther M, et al. The American Society of Hematology 2011 evidence-based practice guideline for immune thrombocytopenia. Blood 2011; 117: 4190-4207.

40. Yacobovich J, Revel-Vilk S, Tamary H. Childhood immune thrombocytopenia-who will spontaneously recover? Semin Hematol 2013; 50: 71-74.
41. Grimaldi-Bensouda L, Nordon C, Leblanc T, et al. Childhood immune thrombocytopenia: A nationwide cohort study on condition management and outcomes. Pediatr Blood Cancer 2017; 64: e26389.

42. Carson KR, Evens AM, Richey EA, et al. Progressive multifocal leukoencephalopathy after rituximab therapy in HIV-negative patients: a report of 57 cases from the Research on Adverse Drug Events and Reports project. Blood 2009; 113: 4834-4840.

43. Pasquet M, Aladjidi N, Guiton C, et al; Centre de Référence National des Cytopénies Auto-immunes de l'Enfant (CEREVANCE). Romiplostim in children with chronic immune thrombocytopenia (ITP): the French experience. Br J Haematol 2014; 164: 266-271.

44. Seidel MG, Urban C, Sipurzynski J, et al. High response rate but short-term effect of romiplostim in paediatric refractory chronic immune thrombocytopenia. Br J Haematol 2014; 165: 419-421.

45. Imbach P, Mark Crowther M. Thrombopoietin-Receptor Agonists for Primary Immune Thrombocytopenia . N Engl J Med 2011; 365: 734-741.

46. Ministerstwo Zdrowia: Leczenie pediatrycznych chorych na przewlekłą pierwotną małopłytkowość immunologiczną (ICD-10 D69.3); https://www.gov.pl/zdrowie/choroby-nieonkologiczne. 2018, 2020.

47. Al-Samkari H, Grace RF, Kuter DJ. The role of romiplostim for pediatric patients with immune thrombocytopenia. Ther Adv Hematol. 2020; 11: 2040620720912992.

48. Vianelli N, Palandri F, Polverelli N, et al. Splenectomy as a curative treatment for immune thrombocytopenia: a retrospective analysis of 233 patients with a minimum follow up of 10 years. Haematologica 2013; 98: 875-880.

49. Leo CA, Pravisani R, Bidinost S, et al. Postsplenectomy recurrence of idiopathic thrombocitopenic purpura: role of laparoscopic splenectomy in the treatment of accessory spleen. G Chir 2015; 36: 153-157.

50. Nomura S. Advances in diagnosis and treatments for immune thrombocytopenia. Clin Med Insights Blood Disord 2016; 9: 15-22. 